\title{
137 DIAGNOSIS AND MANAGEMENT OF FAMILIAL HYPERCHOLESTEROLAEMIA IN A TERTIARY CARDIAC CENTRE
}

C A Taylor, M H Wong, M Curtis, M Taylor, A-M Doherty, R S Patel, C Dollery The Heart Hospital

doi:10.1136/heartjnl-2013-304019.137

Introduction Familial hypercholesterolaemia $(\mathrm{FH})$ is a treatable risk factor for premature myocardial infarction (MI), yet it remains poorly diagnosed in the UK. Current NICE guidance (2008) recommends use of Simon Broome (S-B) criteria for diagnosis of 'definite' or 'possible' FH with referral to a specialist for confirmation of diagnosis and initiation of cascade testing. Cardiac centres have valuable opportunities to identify such patients and implement these recommendations. We examined diagnosis and referral patterns for $\mathrm{FH}$ in light of NICE guidance at our tertiary centre.

Methods Using hospital electronic records we identified all patients with a total cholesterol (TC) $\geq 7.5 \mathrm{mmol} / 1$, who presented to our cardiac services from January to December 2011. For the visit relating to the high TC measurement, we examined patient records to determine if they were assessed by a physician and if there was sufficient documentation to apply S-B criteria, namely (1) family history of premature MI or FH, (2) physical examination for $\mathrm{FH}$ stigmata or (3) consideration of a diagnosis of $\mathrm{FH}$ with referral to a lipid clinic.

Results We examined records for 126 patients and excluded 45 who had not directly seen a physician relating to the index TC measurement. Among the 81 patients included in this study (mean age 57.8, 56\% male), approximately half were seen as in-patients. A total of $4 \%(n=3)$ were classified as 'definite' FH (2 had a prior diagnosis and 1 was not referred), $16 \%(n=13)$ as 'possible' (none referred), and $7 \%(n=6)$ as 'other known lipid disorders'. Over $2 / 3(n=59)$ could not be classified due to insufficient information available to apply the S-B criteria, although 4 of these patients were referred for a specialist opinion and 3 were known to the lipid clinic. Documentation of examination for $\mathrm{FH}$ stigmata was absent in almost all cases $(98 \%, n=79)$. Family history of coronary heart disease (CHD) was documented in $67 \%$ $(n=54)$ of cases, of whom $54 \%(n=29)$ had a positive history. Of the latter, the type of $\mathrm{CHD}$ and age of onset were adequately documented in only $69 \%$ and $66 \%$ respectively. Documentation of family history of high cholesterol was absent in $89 \%$ of cases. In $43 \%(n=35)$ of all cases there was no documentation that the cholesterol result had been noted. Finally we estimated that approximately $4-6 \%$ of acute coronary syndrome patients had TC levels $>7.5$, thus representing a potentially enriched population for $\mathrm{FH}$.

Conclusions While a significant opportunity exists in tertiary cardiac units to identify and refer potential FH cases, S-B criteria are not being routinely documented to consider such patients for onward referral. Universal focus on high dose statin therapy after ACS may in part have contributed to diminished consideration of 
the wider implications of FH and familial risk. Raising awareness of $\mathrm{FH}$ in cardiac units across the country could have significant population health benefits through cascade screening. 